\title{
LA CIUDAD DE ZARAGOZA EN UN ESCENARIO DE CRISIS: DIAGNÓSTICO Y PROPUESTAS TERRITORIALES PARA NUEVOS PARADIGMAS URBANOS ${ }^{1}$
}

\author{
José Luis Calvo Palacios, Ángel Pueyo Campos y María Zúñiga Antón \\ Dpto. de Geografía y Ordenación del Territorio \\ Universidad de Zaragoza
}

\begin{abstract}
Resumen: La crisis del modelo económico está llevando a las grandes ciudades españolas a replantearse sus modelos de crecimiento y de gestión. Este artículo hace una consideración de cuál podría ser el proyecto urbano para la ciudad de Zaragoza en las próximas décadas.
\end{abstract}

Palabras clave: Zaragoza, Gran Recesión, paradigmas urbanos, planificación urbanística, ciudad compacta.

\begin{abstract}
The crisis of the economic model is leading Spanish cities to rethink their growth models and management. This article gives an account of what might be the urban project for the city of Zaragoza in the coming decades.
\end{abstract}

Keywords: Zaragoza, Great Recession, urban paradigms, urban planning, the compact city.

Recibido: 2-11-10. Aceptado: 2-2-12

${ }^{1}$ Este artículo se ha realizado dentro de los proyectos de investigación CSO2010-16389 del Programa nacional de I+D+I y del convenios entre Ebrópolis y la Universidad de Zaragoza para la realización de actividades de investigación. 


\section{Introducción}

La actual gran recesión socioeconómica ha puesto en entredicho gran parte de los modelos y políticas urbanas neoliberales de los últimos treinta años. Es un periodo de reflexión, debate y búsqueda de nuevos paradigmas que respondan y den solución a las ineficiencias sociales y espaciales de las ciudades contemporáneas.

Para superar estos momentos de incertidumbre, que se van a prorrogar durante toda esta década, las grandes urbes españolas están reconsiderando sus políticas territoriales, sus modelos de gestión y la continuidad de sus grandes proyectos.

En este contexto se abre un debate en la ciudad de Zaragoza sobre cuál ha sido su trayectoria urbana, cuáles son sus puntos fuertes y limitaciones, cómo ha de reorientar su proyecto de ciudad, y en qué medida los nuevos modelos de planificación física y estratégica tienen que considerar, bajo una propuesta de geometría variable, otros paradigmas urbanos y sus relaciones con su entorno metropolitano.

\section{El espacio urbano es la herencia de un pasado que se proyecta en un presente que cimenta el futuro}

Cuando desde la planificación se piensa en cómo han de plantearse las políticas territoriales siempre hay que considerar el diálogo que se configura en el espacio urbano por la transcendencia de su pasado, que se proyecta en su presente, y que es el que está cimentando la nuevas actuaciones e intervenciones que determinarán el futuro de la ciudad. Esta sucesión consecutiva y continua produce importantes cambios cualitativos y cuantitativos en el espacio urbano, en las actividades y en las relaciones sociales, tanto en la ciudad central como en los municipios circundantes (Sénécal y Bherer, 2009).

Por ello, antes de enunciar nuevas propuestas territoriales conviene conocer la evolución de la ciudad para diagnosticar su situación, considerando sus antecedentes, puntos fuertes y limitaciones. En el caso concreto de la ciudad de Zaragoza es importante señalar tres grandes periodos cuyas improntas socioterritoriales van a influir en la definición de las futuras políticas urbanas:

- 1960-1980: la configuración de la ciudad compacta contemporánea.

- 1980-2008: el desacoplamiento entre el crecimiento urbano y la demanda demográfica.

- 2008-2011: el contexto presente de la Gran Recesión con su incertidumbre política, social, y económica. 


\subsection{0-1980: la configuración de la ciudad compacta contemporánea}

La ciudad de Zaragoza no ha seguido el modelo de crecimiento de las ciudades maduras que explicaba la Ley de Clark (Clark, 1951) ya que no se produce una disminución sucesiva de las densidades desde el centro hacia las periferias. La crisis del núcleo histórico central propició el crecimiento de sus densidades hacia las orlas exteriores.

Este modelo explicaría la evolución de la ciudad en los años sesenta con la saturación inicial y paulatino vaciado del centro histórico en detrimento de barrios, entonces periféricos, como San José, Las Fuentes, Oliver, Valdefierro, Delicias, etc. (Calvo, 1980).

Este proceso responde al movimiento de invasión-sucesión que modifica los planteamientos de Clark, y que recoge algunos de los enunciados treinta años antes por Burgess sobre el crecimiento urbano en círculos concéntricos. Para la ciudad de Zaragoza, como ya ese explicaban en las investigaciones de Calvo, se podría hablar de una adaptación de este modelo territorial con altas densidades en la periferia de la ciudad siguiendo las avenidas de salida hacia las carreteras de Valencia, Castellón, Barcelona, Huesca, Logroño y Madrid. Los estrangulamientos que suponían los cursos fluviales y las trincheras del ferrocarril, o posteriormente las autopistas de entrada a la ciudad, generaron crecimientos en forma de pata de ganso a modo de pseudópodos que rompían el modelo anular que propugnaba Burgess (Calvo, 1980; Park y Burgess, 1925). Este proceso se mantendrá en décadas posteriores con los nuevos barrios de la margen izquierda y en los proyectos entre el Segundo, Tercer y el Cuarto Cinturón de circunvalación.

Todo esto sucedía en un momento en el que había que dar respuesta a las necesidades de una ciudad que, si en 1960 contaba con 303.975 habitantes, diez años más tarde alcanzaba los 469.366 residentes, en la que ha sido la fase de mayor crecimiento de toda su historia, y que ha respondido al cambio social y territorial de una España que dejó de ser rural para convertirse en urbana. Conviene señalar que esta ocupación de nuevos espacios fue proporcional a su incremento demográfico, pero presentaba graves problemas por la falta de una planificación de lo que se denominará la "ciudad real de Zaragoza" más allá de sus límites administrativos, que nunca se ha sabido tratar adecuadamente y que es unos de sus cuellos de botella para encauzar la política territorial de la ciudad y su entorno metropolitano.

En el momento actual, estos distritos se están vaciando de la población local a favor de los nuevos desarrollos urbanísticos creados a partir de los años ochenta hasta el presente: ACTUR, los antiguos polígonos industriales de la margen izquierda, los espacios de huerta entre el Segundo y Tercer Cinturón, Parque Goya I y II, Santa Isabel, Valdespartera, Parque Venecia, Arco Sur, etc; así como a las nuevas promociones y urbanizaciones de los núcleos de su entorno metropolitano como Casetas, 
Utebo, Cuarte, Cadrete, María de Huerva, La Puebla de Alfinden, Alfajarín, La Muela, El Burgo de Ebro, Villamayor de Gállego, Villanueva de Gállego, Zuera, La Almunia, Pedrola, etc.

Por lo tanto, estos barrios entonces periféricos, ahora tradicionales, son parte del espacio central de la ciudad de Zaragoza, y teniendo en cuenta las propuestas de planificación estratégica que se están aplicando en ciudades europeas o norteamericanas, tendrían que ser piezas claves en su desarrollo territorial en un horizonte de diez a veinte años. Son espacios sensibles que pueden sufrir los efectos de una degradación y segregación socioespacial si no se actúa con decisión y dinamismo (Ascher, 2005). Por el contrario, con imaginación y disposición podrían ser el germen de un nuevo modelo territorial que responda a la sostenibilidad, la cohesión social, la modernidad y la calidad de vida (Encina et al., 2007).

\subsection{0-2008: el desacoplamiento entre el crecimiento urbano y la demanda demográfica}

En cualquier caso, con la relativa estabilidad demográfica de los años ochenta y noventa Zaragoza sólo creció en veinte mil habitantes. Con la finalización del babyboom, y la casi nula recepción de inmigración tanto interna como extranjera, comienza a mostrar los rasgos de un nuevo diseño urbano en el que la urbanización consume espacio en proporción muy superior a la del incremento de las cifras poblacionales de la capital. Así, en la década de los noventa, ya se apreciaban síntomas que evidenciaban la nueva dimensión de una "ciudad real de Zaragoza", en la que los municipios del entorno crecían en mayor proporción que la metrópoli, que se iban llenando de población más joven en tanto que la ciudad central envejecía (mientras el crecimiento acumulativo de Zaragoza para este periodo fue de un $0,57 \%$, en su entorno metropolitano lo hacía al 3,27\%). El aumento de la esperanza y calidad de vida, el cambio en la organización familiar -con un menor número de efectivos debido a la no convivencia en el mismo hogar de hasta tres generaciones-, y las mejoras económicas auspiciaron un crecimiento en la demanda de las unidades residenciales sin que progresase de manera importante la población, pues al disminuir el tamaño del hogar medio, aumentó substancialmente su número y con él la demanda de nuevas viviendas.

Desde el Gobierno autónomo se dictaban leyes diversas: Ley 10/1993, de 4 de Noviembre, de Comarcalización de Aragón; Ley 8/1996, de 2 de Diciembre, de Delimitación Comarcal de Aragón, seguidas de toda una batería normativa por la que se crean 33 comarcas, de las cuales la 17, Zaragoza ni siquiera llega a constituirse. Esto se debe a las dificultades reales de ponerle puertas y límites a un problema de desarrollo urbano y metropolitanización funcional al que la delimitación de la 
comarca como ente territorial en cuanto tal, lejos de solucionar algún problema, lo que hace es constituirse en otro nuevo, si no en el principal y que va a condicionar una situación presente de crecimiento e ineficiencia metropolitana.

Por ello, aunque Zaragoza llevaba una dinámica mucho más acelerada y vanguardista que los municipios de su entorno, la autonomía municipal de cada uno de estos últimos ha llevado a planteamientos urbanísticos que invalidan desde sus raíces las actuaciones plasmadas en la Planificación Urbana de Zaragoza, con desórdenes tan evidentes como las decenas de miles de viviendas admitidas en los Planes Generales de muchos de los municipios del entorno (las inicialmente previstas 10.000 viviendas de la inacabada Ciudad Zaragoza Golf en La Muela sería uno de los ejemplos más claros).

Pero la ciudad central tampoco eludió grandes recalificaciones de suelo con planes residenciales faraónicos con al menos cinco cifras -es el caso de Arco Sur, con veintiúnmil, o la recalificación Área 2000 del proyecto de San Gregorio y en el municipio de Villanueva de Gállego respectivamente con otras diez mil-, entrando en contradicción con nuevas propuestas territoriales en las que se propugna la regeneración de los espacios centrales y de los barrios tradicionales (Gueyffier y Comte, 2008).

Es cierto que este modelo de urbanismo, desde los finales de los noventa hasta el 2007, ha supuesto la entrada de importantes recursos y plusvalías para todas las arcas municipales procedentes del porcentaje de suelo que la ley le permite, y por toda la tributación asociada a estos desarrollos. Pero estos beneficios iniciales no contemplan para el conjunto de la ciudad -en el actual escenario de Gran Recesión más restrictivo y sin las alegrías presupuestarias anteriores- el mantenimiento económico de unos nuevos espacios en los que se obliga a dotar y/o mantener transporte, agua, vertidos, espacios verdes, equipamientos, etc., y todo ello en una proporción muy superior a lo que él músculo de la ciudad -sus habitantes- demandaban (Borja, 2011).

Paradójicamente este modelo de planificación urbana ha contradicho muchos de los objetivos y reflexiones territoriales expuestas en el PGOUZ de 2007, como:

- Un modelo concentrado como punto de partida, manteniendo sus ventajas de modo de vida, transporte colectivo, complejidad o vida de relación.

- Una ciudad central bien acabada y autocontenida en su expansión en el territorio.

- Unas centralidades periféricas reforzadas, fuertes y bien dotadas.

- Un mayor equilibrio territorial y una mayor "sustentabilidad".

- Una cuidadosa valoración de los elevados costes públicos de las infraestructuras.

- Una descentralización en la escala metropolitana. 
La realidad ha sido divergente a estos objetivos territoriales del PGOUZ, no sólo porque no se han cumplido, sino que se han generado unos lastres e hipotecas estructurales en el crecimiento de la ciudad que van a condicionar muchas de las propuestas y actuaciones a futuro.

\subsection{8-2011: el contexto de la Gran Recesión con su incertidumbre política, social, y económica}

No hay que olvidar que la apuesta de los últimos años supuso reorientar el tejido productivo a través de la logística y el conocimiento, reforzando las actividades industriales (automóvil, papel, electrodomésticos, bienes de equipo, etc.), potenciando los sectores emergentes (renovables, aeronáutica, TIC) o el comercio (grandes centros comerciales como Puerto Venecia, Plaza Imperial, etc.); también se aprovechó el gran evento de la Expo Zaragoza 2008 para transformar y regenerar una parte importante de la ciudad.

Como consecuencia directa se ha intervenido en la ciudad central, se han recuperado las márgenes de sus ríos, el entorno de la Estación Intermodal, y creado o ampliado grandes polígonos industriales dentro y fuera de los municipios (Plataforma Logística de Zaragoza -PLA-ZA-, Empresarium, Polígono Tecnológico de Reciclaje -PTR-, Mercazaragoza, Centro Vía, etc.), con unas expectativas de suelo productivo que superan con mucho las propuestas de crecimiento en el presente escenario de crisis, y con un horizonte incierto hasta los inicios de 2020 (Borja, 2011).

Por ello, las nuevas propuestas territoriales han de primar un modelo no expansivo de la ciudad (Charmes, 2011; Gueyffier y Comte, 2008) estimando las incógnitas sociales y económicas actuales que podrían poner en peligro tanto su reorientación económica como su proyección internacional (Brenner, 2011), y considerando los efectos colaterales de la Gran Recesión sobre el presente de Zaragoza como son:

- Los lastres de una expansión inmobiliaria y una propuesta de crecimiento urbano sin límite aparente en todo el espacio metropolitano, que nunca tuvo en consideración las posibilidades de una crisis y los frenos de unos problemas estructurales que se arrastraban de décadas anteriores (Borja, 2011).

- El modelo de gestión y los déficits crónicos que los poderes públicos, financieros y económicos como impulsores de este modelo de expansión urbana han dejado a la ciudad y a los municipios de su entorno. No hay que olvidar que se contó con la aquiescencia de unos ciudadanos arrullados por la promesa de una bonanza económica ininterrumpida, que no consideraron que el coste de las infraestructuras, equipamientos y servicios estaban siendo financiados por unas plusvalías que no podían ser infinitas. 
Hoy, con el cambio de las condiciones económicas y sociales, esos mismos ciudadanos contemplan un contexto social en el que se abren los fantasmas del paro, la desigualdad, la exclusión social y la marginalidad (Chauvel, 2008) en una ciudad que siempre se ha caracterizado por una clase media-baja muy homogénea, con escasa polarización de las rentas, y unos altos niveles de seguridad ciudadana.

\section{Condicionantes e implicaciones socioterritoriales de la Gran Recesión para la ciudad de Zaragoza}

Como la mayoría de las metrópolis españolas, Zaragoza ha seguido un modelo territorial fundamentado en las plusvalías urbanísticas que no se ha correspondido con el crecimiento de la población o su actividad económica, ni ha considerado otras propuestas urbanas más sostenibles como la rehabilitación o el crecimiento compacto, que sí se explicitaban en el PGOUZ.

Esta expansión acelerada se ha frenado radicalmente en los últimos dos años y medio paralizando muchos proyectos urbanísticos ya aprobados y con incertidumbres para otros muchos como Arco Sur, Empresarium, PTR, etc.

La consecuencia es que tras tres décadas de expansión urbana en España, y en Zaragoza en particular, se ha producido una acumulación de las plusvalías en una minoría, sin diversificar sus inversiones en otras actividades económicas salvo las derivadas de otras operaciones de suelo (Borja, 2011).

No se tuvieron en cuenta los condicionantes de la expansión urbana en términos de sostenibilidad o de costes en los desplazamientos para atender a una población cada vez más dispersa y cada vez más segmentada de acuerdo con su nivel socioeconómico (Encina et al., 2007; Fariña y Naredo, 2010). Todo ello conlleva implicaciones e hipotecas territoriales a futuro para Zaragoza como son:

- Un espacio periférico inacabado más grande que la ciudad central, con déficits en comunicaciones, transporte público, infraestructuras y equipamientos que van a ser difícilmente solucionados en igualdad de condiciones que otros barrios tradicionales. La contracción de la actividad económica supone la existencia de un capital urbano inmovilizado con miles de viviendas nuevas sin vender, más de cuarenta mil viviendas vacías y las utilizadas por los octogenarios van a condicionar la evolución de la ciudad en la próxima década.

- El mantenimiento de proyectos de expansión urbana que no responden a las perspectivas de crecimiento demográfico y económico real (que se va a agravar con la colmatación de barrios ya construidos como Valdespartera o la llegada de residentes a Arco Sur o Parque Venecia, o los procesos de traslado desde los 
polígonos industriales tradicionales a los nuevos), y que va a suponer la hipoteca de los barrios tradicionales con áreas residenciales obsoletas.

- Un desplazamiento y concentración de los jóvenes en las nuevas zonas urbanas de la periferia, con un modelo de vida y relación más centrados en las grandes superficies comerciales que en el espacio público, agravando los problemas para el desarrollo y evolución de los barrios más tradicionales en los que se concentra la población envejecida y los emigrantes con menores recursos.

- Un modelo urbano con altas densidades en las orlas intermedias y que se ha vaciado en las partes más deterioradas de la ciudad consolidada, con barrios tradicionales que han perdido su identidad y heterogeneidad social, que se han debilitado y vaciado del tejido comercial -y de los servicios privados de calidadpor la salida de las generaciones más jóvenes y con mayores recursos.

- Un fuerte envejecimiento de la población que no se está abordando con realidad y generosidad, y al que habrá que dar una respuesta de manera decidida en la próxima década.

- Un aumento de los gastos corrientes y de mantenimiento de los servicios infraestructuras y espacios públicos en una ciudad que ha duplicado su superficie sin que se incremente su población, y con unas mayores cargas sociales por los efectos directos de la crisis económica.

- Una incipiente consolidación de marginalidad y de los guetos sociales por la concentración de ciertos grupos de población en las zonas urbanas más envejecidas y en las que la intervención urbana ha sido poco sensible a sus problemas En cierta medida se podría consolidar situaciones similares a las de la ciudad histórica en los años setenta y ochenta del siglo pasado.

\section{Cambios de los paradigmas urbanos y principios territoriales que han de modificar el modelo de ciudad}

Por ello, en este momento de incertidumbre socioeconómica y deriva política es cuando se debe reflexionar y considerar que frente al modelo de ciudad neoliberal (Fitoussi et al., 2004) hay que explorar otros paradigmas y propuestas de ciudad recuperando el modelo mediterráneo de urbanismo compacto, con cierta densidad (Brugel, 2006; Delbaere, 2010; Halbert, 2010), mezcla de usos y grupos sociales, y mínimo desplazamiento (Friedmann, 2011). Un modelo de ciudad cuyo eje central quiere ser el espacio público (Guilliéron, 2010; Charmes, 2011; Galès, 2011) y en la que se deben considerar los valores éticos y sociales para nuevas políticas territoriales eficientes y valientes que pongan en cuestión estos años de especulación inmobiliaria y expansión sin límites (Borja, 2011; Brenner, 2011), y que algunos autores 
incluso plantean en escenarios de contraliberalización radical (Lefebvre, 1972; Harvey, 2003).

Para Zaragoza, teniendo en cuenta este cambio de paradigma, algunos de los principios territoriales que podrían marcar las futuras propuestas en su desarrollo urbano serían (Muso 2008; Kriken y Enquist, 2010):

- El mantenimiento de la densidad y compacidad, con diversidad morfológica y variedad tipológica, que garanticen la compatibilidad en los usos del suelo, la viabilidad de los servicios, las redes, y en especial el transporte público.

- La renovación y reconstrucción de los espacios obsoletos o degradados, evitando la desintegración del espacio urbano y que se generen incomprensiones, inseguridad y marginalidad que irán en detrimento de la ciudad y su ciudadanía.

- La sostenibilidad, como compromiso ético con el medio ambiente, regenerando el territorio por medio de corredores verdes y sistemas de espacios abiertos, minimizando los desplazamientos intraurbanos e intrametropolitanos, pero garantizando una buena accesibilidad merced al transporte público, el desplazamiento a pié o el vehículo privado de bajo impacto -bicicleta, vehículo eléctrico, compartido, etc.-

- El refuerzo de la identidad, de la pertenencia y del sentido del lugar, fomentando el arraigo y la integración del foráneo, garantizando la diversidad y la tolerancia, y construyendo espacios de pluralidad que consideren los procesos de invasión-sucesión.

- El fomento de espacios de productividad y diversidad económica aprovechando la especialización sectorial propugnada en la década anterior, y la implementación de las tecnologías de la información y de la comunicación para crear territorios aumentados.

- Un modelo de gestión contenido en el gasto, en el que se impulsen propuestas creativas soft o de bajo coste y que impliquen responsablemente a la ciudadanía.

Por lo tanto, en este momento de cambios de paradigmas y de nuevos principios territoriales, es obligado que Zaragoza y su entorno se articulen en un modelo de geometría variable que supere el encorsetamiento comarcal (Pascual, 2002) para favorecer la organización más allá de los límites de la capital regional. Se ha de conseguir un consenso que aúne a la ciudadanía, las fuerzas políticas, las administraciones públicas y el tejido empresarial creando un espacio metropolitano de calidad, próximo, plural, amigable, sostenible, cohesionado socialmente y con impulso económico (Vanier, 2008).

Debería ser un espacio en el que converja la tradición industrial y comercial, con la innovación de la logística y de las actividades ligadas al conocimiento para crear 
un territorio aumentado (Vergara y De las rivas, 2004; Muso, 2008). Hay que retornar a una ciudad de alta calidad en la vida local para hacerla fuerte en el contexto global (Sort, 2006). Además de las consideraciones sectoriales y de oportunidad, hay que pensar en actuaciones que articulen el territorio, busquen el bien común y que se planifiquen y desarrollen más allá de los plazos convencionales del ejercicio político. Lamentablemente, una parte del desarrollo urbano anterior obvio el carácter unitario de la ciudad en aras de un lucro económico y político, que no ha valorado las verdaderas necesidades del ciudadano y que ha postergado la financiación de las inversiones a las generaciones futuras.

Habrá que renunciar a los grandes proyectos emblemáticos que estén simplemente basados en afanes recaudatorios o que carezcan de una conexión directa con las necesidades del ciudadano y que sean un impulso socioeconómico que beneficie estratégicamente a la ciudad. El modelo territorial futuro ha de dimensionar los crecimientos en función de las necesidades (Castellett y D'Acunto, 2006), siempre teniendo en cuenta que aún para mantener mejoras evidentes hace falta una solidez financiera de la que ahora mismo se carece. Así, toda propuesta territorial tendrá que considerar previamente:

- La valoración cuidadosa de las inversiones evitando los desequilibrios presupuestarios.

- La necesidad de una gobernanza territorial en la que sea obligado el consenso gobierno (Estado-Región-Provincia-Municipio) y sociedad (agentes sociales-ciudadanía-instituciones públicas), por lo menos en los grandes temas de redes o en su implicación sobre las mismas (Pascual, 2002).

- La obligada convergencia entre la Planificación Estratégica y la actuaciones derivadas de la Planificación Real (PGOUZ, directrices territoriales, planes sectoriales metropolitanos, regionales, comarcales, etc.)

- La conciliación de la planificación a largo plazo, las actuaciones inmediatas y las modificaciones puntuales que se imponen por los avatares en una sociedad contemporánea globalizada (Charmen, y Souami, 2009).

\section{Propuestas territoriales para Zaragoza atendiendo a los nuevos paradigmas urbanos}

La necesidad de recuperar la austeridad en el consumo del territorio ha de servir para contextualizar las propuestas territoriales acordes con los principios anteriormente expuestas abordando con decisión cambios, comenzando por temas tan obvios como: 
- La divergencia entre el crecimiento de las variables demográficas y el de las viviendas programadas.

- La capacidad del suelo urbano calificado y sus desarrollos futuros.

Es el momento para realizar propuestas que acaben con las diferencias entre la ciudad real y la ciudad planificada, considerando el área metropolitana como un ente territorial de geometría variable para crear un espacio coherente de sostenibilidad, evitando la dispersión y la desorganización de las dos últimas décadas. Se trataría, en esencia, de construir un espacio de futuro en la que la globalidad se combine con lo local, integrando la vanguardia, la sostenibilidad y la identidad de una Zaragoza bimilenaria, reconociendo las limitaciones presupuestarias para la próxima década.

A modo de conclusiones, y teniendo en cuenta la interacción entre el pasado y el presente, se plantean los siguientes objetivos territoriales para la Zaragoza del futuro:

- Una ciudad compacta pero policéntrica que se apoye en los grandes barrios y en los núcleos de su entorno metropolitano, en la que se concilie la proyección global e internacional con los debates y problemas locales (Hubert, 1993), en la que se favorezca el acceso a los equipamientos públicos de calidad, donde se minimicen los desplazamientos urbanos y se apueste por el transporte colectivo o los modelos de desplazamiento blando -bicicleta o a pié- (Pozueta et al. 2010).

- Un espacio urbano en el que exista compatibilidad y diversidad de usos, que favorezca la mezcla y la variedad tipológica de equipamientos, actividades y grupos sociales, y que recupere el espacio público como espacio de vanguardia, relación, identidad y modernidad (Kirszbaum, 2008; Smith, 2011).

- La adecuación del crecimiento urbano a las previsiones demográficas y productivas, priorizando la renovación y reconstrucción del espacio heredado o consolidado, en especial los barrios que crecieron en la segunda mitad del siglo pasado (Gueyffier y Comte, 2008).

- Un urbanismo próximo y humano en el que el barrio tradicional sea el motor de la innovación, de la sostenibilidad y de la integración, potenciando sus puntos fuertes y revitalizando los ejes funcionales, los espacios de relación, la pluralidad de actividades o el comercio de proximidad. (Estèbe, 2008).

- Una adaptación de la ciudad a las nuevas relaciones espacio-temporales integrando la cuarta dimensión digital y la emergencia de una nueva lógica ciberurbana que fomente la urbiótica y el desarrollo de un territorio aumentado (Mitchell, 2001; Muso, 2008; Vivant, 2009).

- El fomento de la sostenibilidad con un compromiso ético con el medio ambiente, en el que se minimice el desplazamiento y se asegure una movilidad (Röhr, 2009; Pozueta et al. 2010) y que acerque las zonas verdes a los espacios residencias (Delbaere, 2010). 
- Una metrópoli para las personas, que valore la diversidad, la emigración y los ancianos como vectores para la economía local; en la que se minimicen las distancias culturales entre los ciudadanos y se erradique la emigración de las generaciones más competitivas (Fitoussi, 2004; Friedmann, 2011; Galès, 2011; Smith, 2011).

- Una ciudad en las que se consideren a los valores ciudadanos como vector para construir ciudad: tanto para mejorar y desarrollar la ciudad con menores costes socioeconómiocos como para desactivar los riesgos de quiebra, confrontación y segmentación sociales en el espacio urbano.

\section{Bibliografía}

Ascher F.(2005). Los nuevos principios del urbanismo. Madrid, Alianza ensayo.

Borja, J. (2011). ¿Un cambio de ciclo o un cambio de época? Siete líneas para la reflexión y la acción. Urban, 1, pp. 83-88.

Brenner, N. et al. (2011). ¿Y después de la neoliberalización? Estrategias metodológicas para la investigación de las transformaciones regulatorias contemporáneas. Urban, 1, p. 21.

Burgel, G. (2006). La revanche des villes. Paris, Hachette.

Clark, C. (1951). Urban population demities. Journal of the Royal Statistical Society, vol. 114, pp. 490-496.

Calvo Palacios, J.L. (1980). Unidades de análisis y densidades urbanas: Zaragoza. Geographicalia, ํㅡㄴ 5, pp. 5-32.

Castellett, M y D'Acunto, M. (2006). Marketing per il Territorio. Srategie e politiche per los sviluppo locale nell'economica globalizzata. Milano, Franco Angeli.

Charmen, E. y Souami, T. (2009). Villes revées, villes durables. Paris, Gallimard.

Charmes, E. (2011). Densité, formes urbaines et villes durables. En Damon, J. (ed.) Villes à vivre. Modes de vie urbains et défis envi- ronnementaux. Paris, Odile Jacob, pp. 8199.

Chauvel, L. (2008). La globalcity et ses critiques. Tensions dans l'espace urbain et divisions des classes moyennes. En Damon, J. (ed.) Vivre en ville. Observatoire mondiale des modes de vie urbains. Paris. PUF, pp. 161-181.

Churchill, H.S. (1945). The city is the people. New York, Reynal\&Hichcok.

Delbaere, D. (2010). La fabrique de l'espace publique. Paris, Ellipses.

Dubois-Taine, O.P. (2010). Les nouvelles mobilités. Paris, La Documentation Française. Centre d'Analyse Stratégique.

Dupuy, G. (2007). La fracture numérique Paris, Ellipses.

Encina J. et al. (2007). La ciudad a escala bumana. Sevilla, Atrapasueños.

Estèbe, Ph. (2008.) Gouverner la ville mobile. Paris, PUF, coll. La ville en débat.

Fariña, J. y Naredo, J.M. (2010). Libro Blanco sobre Sostenibilidad en el Planeamiento Urbano Español. Madrid, Centro de Publicaciones de la Secretaría General Técnica del Ministerio de la Vivienda.

Farnham, Ashgate, p. 181-198. 
Fitoussi, J.P. et al. (2004). Ségrégation urbaine et intégration sociale. Paris. La Documentation Française. Conseil d'Analyse Économique.

Friedmann, J. (2011). Barrio por barrio: reclamando nuestras ciudades. Urban, 1, pp. 13-19.

Galès, P. (2011). Le rétour des villes européennes. Paris, Presses de la Fondation Nationale des Sciences Politiques.

García Vazquez C. (2004). Ciudad Hojaldre. Barcelona, Gustavo Gili.

Glaesser, E. (2011). Des villes et des hommes enquête sur un mode de vie planétaire. Paris, Flammarion.

Gueyffier, M.F. y Comte, Ph. (2008). Bâtir le futur de l'habitat à partir du parc existant. En Place, J.M. (eds.) Futur de l'habitat. Paris, PUCA, pp. 49-59.

Guilliéron, PY et al. (2010). La navigation pedestre au bénefice de la mobilité piétonne. En Wack, M. et al. (eds.) Géopositionnment et mobilités. Montbéliard, Université de Technologie de Belfort-Montebéliard, pp. 255-296.

Halbert, L. (2010). L'avantage métropolitain. Paris, PUF, coll. La ville en débat.

Harvey, D. (2003). Espacios de esperanza. Madrid, Akal.

Hubert, J.P. (1993). La discontinuité critique. Paris, Publications de la Sorbonne.

Kirszbaum, Th. (2008). Rénovation urbaine. Les leçons américaines. Paris, PUF, coll. La ville en débat.

Kriken, J.L. y Enquist, Ph. (2010). City Building: Nine Planning Principles for the Twenty-First Century. Ed Princeton Architectural Press.

Lefebvre, H. (1972). La revolución urbana. Madrid, Alianza Editorial.
Mitchell, W. J. (2001). E-topía. Barcelona, Ed Gustavo Gili.

Muso, P. (2008). Territoires et cyberspace en 2030. Paris, La Documentation Française, Coll. Travaux

Park, R. E. y Burgess, E. W. (1925). The city the university of Chicago press. Londres, $239 \mathrm{p}$.

Pascual, JM. (2002). La gestión estratégica de las ciudades. Un instrumento para gobernar las ciudades en la era info-global. Sevilla, Junta de Andalucía.

Pozueta, J. et al. (2010). La ciudad paseable. Madrid, CEDEX.

Röhr, Th. (2009). Systèmes universels mobiles d'information voyageur. En Wack, M. et al. (eds.) Géopositionnment et mobilités. Montbéliard, Université de Technologie de Belfort-Montebéliard, pp. 339-361.

Sénécal, G. y Bherer, L. (2009). La métropolisation et ses territoires. Québec, Presses de l'Université du Québec.

Smith, M.P. (2011). Translocality: A Critical Reflection. En Brickell, K. y Datta A. (eds.) Translocal Geographies. Spaces, places, connections.

Sort J. (2006). Redes Metropolitanas. Barcelona, Gustavo Gili.

Vanier, M. (2008). Le pouvoir des territoires Essais sur l'interterritorialité. Paris, Economica.

Vegara A y De las rivas JL. (2004). Territorios inteligentes. Madrid, Fundación metrópoli.

Vivant, E. (2009). Qu'est-ce que c'est la ville créative?. Paris, PUF, coll. La ville en débat. 